\title{
Time-dependent decreases in nucleus accumbens AMPA/NMDA ratio and incubation of sucrose craving in adolescent and adult rats
}

\author{
Danielle S. Counotte • Christopher Schiefer • \\ Yavin Shaham • Patricio O'Donnell
}

Received: 11 June 2013 / Accepted: 11 September 2013 /Published online: 11 October 2013

(C) The Author(s) 2013. This article is published with open access at Springerlink.com

\begin{abstract}
Rationale and objective There is evidence that cue-induced sucrose seeking progressively increases after cessation of oral sucrose self-administration (incubation of sucrose craving) in both adolescent and adult rats. The synaptic plasticity changes associated with this incubation at different age groups are unknown. We assessed whether incubation of sucrose craving in rats trained to self-administer sucrose as young adolescents, adolescents, or adults is associated with changes in 2-amino3-(3-hydroxy-5-methyl-isoxazol-4-yl)propanoic acid (AMPA)/ $\mathrm{N}$-methyl-D-aspartate (NMDA) ratio (a measure of postsynaptic changes in synaptic strength) in nucleus accumbens.

Methods Three age groups initiated oral sucrose selfadministration training (10 days) on postnatal day (P) 35 (young adolescents), P42 (adolescents), or P70 (adults). They were then tested for cue-induced sucrose seeking (assessed in an extinction test) on abstinence days 1 and 21. Separate groups of rats were trained to self-administer sucrose or water (a control condition), and assessed for AMPA/NMDA ratio in nucleus accumbens on abstinence days $1-3$ and 21 .

Results Adult rats earned more sucrose rewards, but sucrose intake per body weight was higher in young adolescent rats. Time-dependent increases in cue-induced sucrose seeking (incubation of sucrose craving) were more pronounced in adult rats, less pronounced in adolescents, and not detected in young adolescents. On abstinence day 21, but not days 1-3, AMPA/ NMDA ratio in nucleus accumbens were decreased in rats that
\end{abstract}

D. S. Counotte $(\bowtie) \cdot$ C. Schiefer $\cdot$ P. O'Donnell

Department of Anatomy and Neurobiology, University of Maryland School of Medicine, 20 Penn St, Rm S-251, Baltimore, MD 21201, USA

e-mail: d.s.counotte@gmail.com

Y. Shaham

Behavioral Neuroscience Branch, IRP-NIDA, Baltimore, MD, USA self-administered sucrose as adults and adolescents, but not young adolescents.

Conclusions Our data demonstrate age-dependent changes in magnitude of incubation of sucrose craving and nucleus accumbens synaptic plasticity after cessation of sucrose selfadministration.

Keywords AMPA/NMDA ratio - Extinction - Incubation of reward craving $\cdot$ Nucleus accumbens $\cdot$ Relapse $\cdot$ Sucrose . Synaptic plasticity

$\begin{array}{ll}\text { Abbreviations } \\ \text { PFC } & \text { Prefrontal cortex. } \\ \text { NAc } & \text { Nucleus accumbens } \\ \text { AMPA } & \text { 2-Amino-3-(3-hydroxy-5-methyl-isoxazol-4- } \\ & \text { yl)propanoic acid } \\ \text { NMDA } & \text { N-methyl-D-aspartate } \\ \text { P } & \text { Postnatal day } \\ \text { EPSC } & \text { Excitatory postsynaptic current }\end{array}$

\section{Introduction}

Many studies indicate that the adolescent brain continues to develop (Blakemore and Robbins 2012; Casey et al. 2010; Counotte et al. 2011). In particular, neurochemical, structural, and electrophysiological evidences suggest that reward-related brain regions and their connections such as prefrontal cortex (PFC) and nucleus accumbens (NAc) mature during adolescence (Brenhouse et al. 2008; Giedd 2004; Tseng and O'Donnell 2007). These age-dependent physiological changes may underlie increased sensitivity to nondrug rewards during the adolescent phase in humans (Crone and Dahl 2012; Van Leijenhorst et al. 2009). An important question is whether reward exposure 
impacts adolescent brain development in a different way and with longer-lasting consequences than the mature adult brain.

Craving is a subjective state that precedes and accompanies reward seeking. Rats trained to self-administer drug rewards (Grimm et al. 2001; Neisewander et al. 2000; Shalev et al. 2001) or a nondrug sucrose (Grimm et al. 2005; Grimm et al. 2002) reward demonstrate time-dependent increases in cueinduced reward seeking during abstinence. This phenomenon is termed "incubation of reward craving" (Grimm et al. 2001; $\mathrm{Lu}$ et al. 2004b). Incubation of reward craving has been primarily demonstrated in two procedures used to assess cue-induced reward seeking (Everitt and Robbins 2005; See 2002): a single extinction session in the presence of the reward-associated cues (Lu et al. 2005; Theberge et al. 2013; Uejima et al. 2007) and discrete-cue-induced reinstatement after extinction of lever presses without these cues (Grimm et al. 2005; Li and Frantz 2010; Marchant et al. 2013).

Despite human evidence of increased vulnerability to addiction following adolescent drug use (Palmer et al. 2009), studies in rats have shown that the magnitude of incubation of cocaine craving is reduced following adolescent cocaine selfadministration ( $\mathrm{Li}$ and Frantz 2009). Additionally, while age differences in incubation of heroin craving were not formally assessed, adolescent heroin self-administration leads to reduced cue-induced heroin seeking after 12 abstinence days (Doherty et al. 2013). In contrast, Li and Frantz reported a lack of age differences in incubation of sucrose craving, as assessed in the discrete-cue-induced reinstatement procedure (Li and Frantz 2010).

The mechanisms underlying incubation of drug (in particular cocaine) craving were examined in many studies (Loweth et al. 2013; Marchant et al. 2013; Pickens et al. 2011). In contrast, much less is known on mechanisms of incubation of sucrose craving, and there are no published data on mechanisms of incubation of sucrose craving in adolescent rats. In adult rats, systemic injections of a mu-opioid receptor antagonist or systemic or central amygdala injections of an mGluR2/3 agonist (resulting in decreased evoked glutamate release) selectively attenuate enhanced cue-induced sucrose seeking during late abstinence, but not lower cue-induced sucrose seeking during early abstinence (Grimm et al. 2007; Uejima et al. 2007). These data suggest a role of brain opioid receptors and central amygdala glutamate in incubation of sucrose craving. There is also evidence that injections of a D1 receptor antagonist into NAc core or shell block cueinduced sucrose seeking during both early and late abstinence (Grimm et al. 2011). These data suggest a general role of NAc D1-family receptors in cue-induced sucrose seeking, independent of the abstinence period.

Synaptic plasticity in the form of time-dependent accumulation of calcium-permeable (CP) GluA2-lacking 2-amino-3-(3hydroxy-5-methyl-isoxazol-4-yl)propanoic acid receptors (AMPARs) in NAc, which results in increased glutamate- mediated synaptic transmission, plays a critical role in incubation of cocaine craving (Conrad et al. 2008; Lee et al. 2013; Wolf and Ferrario 2010). On the other hand, there is evidence that a natural reward (repeated exposure to male sexual behavior) causes a long-lasting reduction in AMPA/ $N$-methyl-D-aspartate (NMDA) ratio in NAc medium spiny neurons, indicating decreased glutamate-mediated synaptic strength and neurotransmission (Pitchers et al. 2012). The effect of sucrose selfadministration on NAc synaptic plasticity and the potential role of this plasticity in incubation of sucrose craving in adolescent or adult rats are unknown.

Here, we first determined cue-induced sucrose seeking in extinction tests after 1 or 21 abstinence days in rats that initiate oral sucrose self-administration during young adolescence (starting self-administration at P35), adolescence (starting at P42), or adulthood (starting at P70). We then determined AMPA/NMDA ratio in NAc after 1-3 or 21 abstinence days in different groups of rats that initiated self-administration of sucrose or water (a control condition) during these different age periods.

\section{Materials and methods}

\section{Animals}

Subjects were 146 male Long-Evans (Charles Rivers Laboratories, USA) that arrived 2 weeks before sucrose selfadministration experiments started. Three age groups were used: young adolescents that started self-administration at P35, adolescents that started at P42, and adults that started at P70. Rats were housed two per cage under reversed lighting conditions (lights on at 7 p.m.). Rats had unlimited access to food and water, except on days before a sucrose selfadministration session, in which case they received $20 \mathrm{~g}$ of chow at the end of the day. All experiments were approved by the University of Maryland School of Medicine Institutional Animal Care Committee and were conducted in accordance with the United States Public Health Service Guide for the Care and Use of Laboratory Animals.

\section{Sucrose self-administration}

Rats were tested in self-administration chambers (Med Associates, St. Albans, VT) housed within sound-attenuating boxes. Each chamber was equipped with two levers located $9 \mathrm{~cm}$ above the floor, a house light, a tone cue, and a light cue. Rats self-administered with a $10 \%$ sucrose solution (or water as a control for the electrophysiology experiments) during 10 daily 3-h sessions. Presses on the active (and retractable) lever resulted in the activation of the infusion pump, and thus the delivery of $0.6 \mathrm{ml}$ of $10 \%$ sucrose solution in the receptacle, accompanied by audiovisual cues (a tone and a light), and the 
house light switching off during the $20 \mathrm{~s}$ time-out, during which active lever presses were recorded but did not lead to the delivery of sucrose (Grimm et al. 2005; Karlsson et al. 2013; Uejima et al. 2007). Presses on the inactive (and stationary) lever had no programmed consequences but were recorded. The rats could earn a maximum of 100 rewards. Twelve rats were excluded from the study because they did not reach the acquisition criteria ( $>10$ active lever presses during the last five sessions). This was not different between the age groups.

\section{Cue-induced sucrose seeking}

The test for incubation of sucrose craving is based on previous work (Uejima et al. 2007). After 10 days of sucrose selfadministration, separate groups of rats remained in their home cages for either 1 day or 21 days. Next, we used six groups of rats $(n=12-16)$ in a between-subjects factorial design that included the factors of age group (young adolescents, adolescents, adults) and abstinence day $(1,21)$ to assess cue-induced sucrose seeking in 1-h extinction on abstinence days 1 or 21 . During testing, active lever presses resulted in the activation of the tone and light cues, but not sucrose delivery; additionally, the house light turned off during the 20 -s periods in which the discrete tone-light cues were delivered. The number of active and inactive lever presses was recorded.

\section{Whole-cell recordings}

\section{Brain slice preparation}

In the electrophysiology experiment, we used 12 groups of rats ( $n=4-6$ per group) in a between-subjects factorial design, using the factors of age group (young adolescents, adolescents, adults), reward type (sucrose, water), and abstinence day $(1-3,21)$. The rats were trained to self-administer sucrose or water (a control condition) for 10 days as described above. Either 1-3 days or 21 days after the last self-administration session, rats were anesthetized with chloral hydrate $(400 \mathrm{mg} /$ $\mathrm{kg}$, i.p.) $15 \mathrm{~min}$ before perfusion with ice-cold artificial CSF (aCSF). The rats in these groups did not undergo extinction tests because previous studies indicate that glutamate synaptic plasticity after prolonged withdrawal from the cocaine is due to past drug exposure and independent of acute cue-induced cocaine seeking during the extinction tests (Conrad et al. 2008; Loweth et al. 2013; Wolf and Ferrario 2010). Brains were quickly removed and placed in ice-cold aCSF oxygenated with $95 \% \mathrm{O}_{2}-5 \% \mathrm{CO}_{2}$ and containing the following (in millimolars): $125 \mathrm{NaCl}, 25 \mathrm{NaHCO}_{3}, 10$ glucose, $3.5 \mathrm{KCl}$, $1.25 \mathrm{NaH}_{2} \mathrm{PO}_{4}, 0.5 \mathrm{CaCl}_{2}$, and $3 \mathrm{MgCl}_{2}, \mathrm{pH} 7.45$ (295$300 \mathrm{mOsm})$. Parasagittal sections $(300 \mu \mathrm{m})$ containing the NAc and afferent cortical fibers were obtained with a vibratome in ice-cold aCSF and incubated in warm $\left(\sim 35^{\circ} \mathrm{C}\right)$ aCSF solution constantly oxygenated with $95 \% \mathrm{O}_{2}-5 \% \mathrm{CO}_{2}$ for at least $45 \mathrm{~min}$ before recording. Whole-cell recordings from medium spiny neurons were made using standard electrophysiological techniques (Gruber et al. 2009).

\section{Whole-cell recordings}

Patch electrodes (7-10 M $\Omega$ ) were obtained from $1.5 \mathrm{~mm}$ borosilicate glass capillaries (World Precision Instruments) with a Flaming-Brown horizontal puller (P97; Sutter Instruments) were filled with a solution containing $0.125 \%$ Neurobiotin and the following (in millimolars): $115 \mathrm{Cs}-$ gluconate, $10 \mathrm{HEPES}, 20 \mathrm{CsCl}, 2 \mathrm{MgCl}_{2}, 0.2$ EGTA, 0.1 spermine, 1 QX-314, 2 MgATP, $2 \mathrm{Na}_{2}$-ATP, and 0.3 GTP, pH 7.25-7.30 (280-285 mOsm). DL-AP5 and picrotoxin were fleshly mixed into oxygenated recording aCSF every day before an experiment. Both control and drug-containing aCSF were oxygenated continuously throughout the experiments. All experiments were conducted at $33-35^{\circ} \mathrm{C}$. Medium spiny neurons were identified under visual guidance using infrared (IR) differential interference contrast video microscopy with a $40 \times$ water-immersion objective (Olympus). The image was detected with an IR-sensitive CCD camera and displayed on a monitor. Whole-cell current-clamp recordings were performed with a computer-controlled amplifier (MultiClamp 700A; Molecular Devices), digitized (Digidata 1322; Molecular Devices), and acquired with AxoScope 9 (Molecular Devices) at a sampling rate of $10 \mathrm{kHz}$. Electrode potentials were adjusted to zero before recording without correcting the liquid junction potential.

\section{Synaptic responses}

Synaptic responses were tested in medium spiny neurons in the NAc core with electrical stimulation of cortical afferents with a bipolar electrode made from a pair of twisted Teflon-coated Tungsten wires (tips separated by $\sim 200 \mu \mathrm{m}$ ) and placed $\sim 500 \mu \mathrm{m}$ away from the recorded neuron, with corticostriatal fiber tracts identified under the microscope connecting both sites (Fig. 3b) (Gruber et al. 2009). Stimulation pulses (0.4-1.0 mA; $0.5 \mathrm{~ms}$ ) were delivered every $15 \mathrm{~s}$. Previous work using currentclamp recordings in this preparation has shown that this stimulation intensity is half the intensity necessary to evoke an action potential (Gruber et al. 2009). All experiments were carried out in the presence of picrotoxin $(10 \mu \mathrm{M})$. Upon breaking into the cell, it was voltage-clamped at $-70 \mathrm{mV}$. For $10 \mathrm{~min}$, input resistance was measured using a single hyperpolarizing step every $15 \mathrm{~s}$ and the cell was discarded when the input resistance changed more than $20 \%$ during these $10 \mathrm{~min}$. After $10 \mathrm{~min}$ of baseline recording, the cell was held at $-80 \mathrm{mV}$ for 10 sweeps to record electrically evoked fast AMPAR-mediated currents (EPSCs). Subsequently, the cell was held at $+40 \mathrm{mV}$ to record fast AMPAR- and slower NMDA receptor (NMDAR)-mediated EPSCs. After $10 \mathrm{~min}$, the NMDAR antagonist DL-AP5 
$(100 \mu \mathrm{M})$ was bath-applied to isolate AMPAR-mediated EPSCs at $+40 \mathrm{mV}$. AMPA/NMDA ratios were determined by dividing the peak of the response at $+40 \mathrm{mV}$ in the presence of DL-AP5 (AMPAR-mediated) by the response $40 \mathrm{~ms}$ following stimulation in the absence of DL-AP5 (NMDAR-mediated). The rectification index $\left(i_{\mathrm{r}}\right)$ was calculated by correcting any potential shifts in the reversal potential values $\left(E_{\text {rev }}\right)$ and computed from the following equation: $i_{\mathrm{r}}=\left[I_{-80} /\left(80-E_{\mathrm{rev}}\right)\right] /\left[I_{+40} /\left(40-E_{\mathrm{rev}}\right)\right]$ (Conrad et al. 2008). For EPSCs, the mean of 10 sweeps was taken. At the end of each experiment, the slices were placed in $4 \%$ paraformaldehyde and processed for DAB staining using standard histochemical techniques to verify morphology and location of the neurons.

Statistical analyses

Data were analyzed by ANOVA using the appropriate withinsubjects or between-subjects designs (see "Results"). In case of statistically significant main effects or interaction effects ( $p$ $<0.05$ ), post hoc comparisons were conducted using Fisher $\mathrm{PLSD}$ or Bonferroni tests. Data are presented as mean $\pm \mathrm{SEM}$.

\section{Results}

\section{Sucrose self-administration}

Data were analyzed separately for number of sucrose reward earned, total active lever presses (reward earned + time-out active lever presses), active lever presses during time-out, inactive lever presses, and sucrose consumption per body weight. The analyses included the between-subjects factor of age group and the between-subjects factor of age group (young adolescent, P35 at start; adolescent; P42 at start; and adult, P70 at start). All age groups acquired stable sucrose self-administration (Fig. 1a, session: $F_{(9,747)}=94.1, p<$ 0.001 ), but there was a difference in number of rewards earned between age groups (age group: $F_{(2,83)}=7.1, p=0.001$ ). Post hoc analysis showed that adult rats earned more sucrose rewards than adolescents $(p=0.041)$ or young adolescents $(p=0.001)$. Adult rats also made more active lever presses (Fig. 1b, lever $\times$ age group: $F_{(2,83)}=11.0, p<0.001$ ) due to increased responding during the 20 -s time-out period (Fig. 1c, session $\times$ age group: $F_{(18,747)}=1.8, p=0.066$, age: $F_{(2,83)}=$ $11.2, p<0.001)$. However, when normalized to their body weight, sucrose intake in young adolescent rats was higher than in adult rats (Fig. 1d, age group: $F_{(2,83)}=5.0, p=0.009$ ). There were no differences between the two adolescent groups.

Cue-induced sucrose seeking

Cue-induced sucrose seeking in the extinction tests was higher after 21 abstinence days than after 1 day, and this effect was more pronounced in rats trained to self-administer sucrose as adults than rats that were trained as adolescents and young adolescents (Fig. 2). The statistical analysis included the between-subjects factors of age group and abstinence day ( $n=12-16$ per group). This analysis demonstrated significant effects of abstinence day $\left(F_{(1,80)}=28.2, p<0.001\right)$ and age group $\times$ abstinence day $\left(F_{(2,80)}=4.9, p=0.01\right)$. Post hoc testing demonstrated significant differences between day 1 and day 21 in the adolescent and adult groups $(p<0.005)$ but not in the young adolescent group $(p=0.29)$.
Fig. 1 Self-administration of liquid sucrose in young adolescent, adolescents, and adult rats. a Number of rewards (i.e., lever presses during the active period) earned by the young adolescent (white circles), adolescent (gray circles), and adult (black circles) rats. b Number of active (circles) and inactive (triangles) lever presses during both the active and the time-out period. $\mathbf{c}$ Number of lever presses during the $20 \mathrm{~s}$ timeout period following delivery of the reward. d Sucrose consumption normalized to body weight. ${ }^{*} p<0.05$, adults different from the two adolescent groups; $\# p<0.05$, adults different from the young adolescent groups
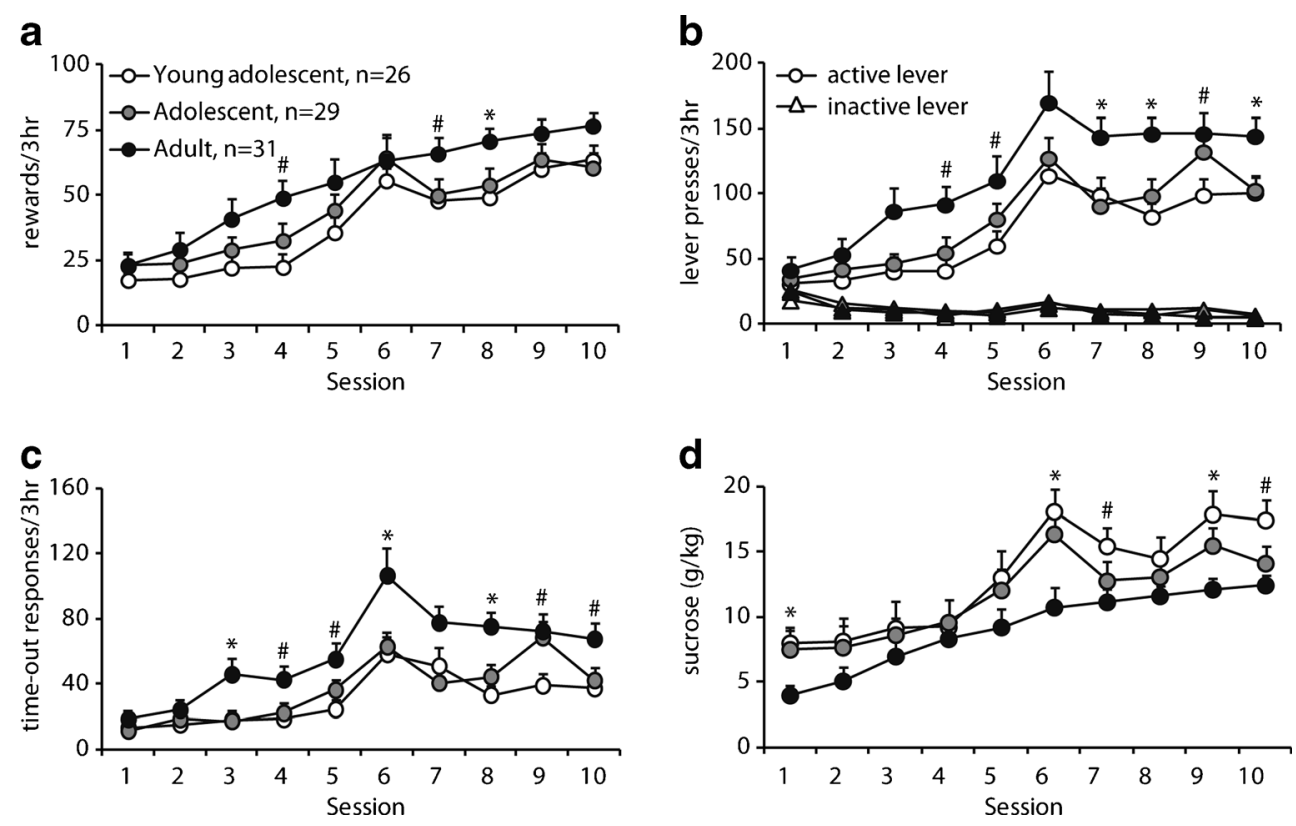
Corticoaccumbens synaptic responses

To determine whether synaptic strength in corticoaccumbens synapses was altered after sucrose self-administration, we measured synaptic currents in medium spiny neurons with electrical stimulation of afferent fibers coming from the PFC in 12 groups of rats ( $n=4-6$ per group). Half of these rats were trained to self-administer sucrose, while the other half selfadministered water (Fig. 3a). The statistical analysis of reward delivery during training included the between-subjects factors of training solution (sucrose, water) and age group (young adolescents, adolescents, adults). This analysis demonstrated significant main effects of age group $\left(F_{(2,54)}=5.8, p<0.005\right)$ and training solution $\left(F_{(1,54)}=75.6, p<0.001\right)$, but no interaction between the two factors. The main effect of age is due to the fact that the adult rats consumed more sucrose and water than the adolescent groups ( $p$ values $<0.05$ ). The relevance of these age differences in training to the age differences to incubation of sucrose craving (above) and the electrophysiology findings described below is unknown.

Following 1-3 days of abstinence, there was no change in AMPA/NMDA ratio or rectification index ( $p$ values $>0.05$; Fig. $3 \mathrm{c}-\mathrm{e}$ ). The AMPA/NMDA ratio was significantly reduced 3 weeks after sucrose self-administration compared to rats that self-administered water (Fig. 3f, g, training solution: $\left.F_{(1,33)}=14.1, p<0.001\right)$. Post hoc analysis showed that this difference was significant in the adolescent and adult groups $(p<0.05)$, but not in the young adolescent group. To determine whether the reduced AMPA/NMDA ratio following sucrose self-administration was due to changes in CPAMPARs, we also assessed rectification of the AMPARmediated response in the same cells by dividing the amplitude of the response at $-80 \mathrm{mV}$ by the amplitude at $+40 \mathrm{mV}$ (Conrad et al. 2008). We did not find differences in rectification in any of the groups (Fig. 3h), suggesting that sucrose self-administration and 21 days of abstinence does not lead to changes in CP-AMPARs in NAc.

\section{Discussion}

We assessed incubation of cue-induced sucrose craving during abstinence and accumbens synaptic plasticity in the form of AMPA/NMDA ratio in rats that initiated oral sucrose selfadministration at P35 (young adolescents), P42 (adolescents), or adults (P70). We found that incubation of sucrose craving was most pronounced in the adult training group, moderate in the adolescent training group, and absent in the young adolescent training group. We also found that decreased AMPA/ NMDA ratio on abstinence day 21 was most pronounced in the adult training group, moderate in the adolescent training group, and very weak in the young adolescent training group. These two sets of correlative data, and the observation that
AMPA/NMDA ratio was unchanged on abstinence days $1-3$, suggest a potential role of accumbens synaptic plasticity in incubation of sucrose craving. However, this correlative data set maybe too preliminary for such a conclusion in the absence of additional experiments demonstrating in adult- and adolescent training groups that restoring AMPA/NMDA ratio on abstinence day 21 decreases "incubated" cue-induced sucrose seeking.

We also found that sucrose consumption per body weight was higher in the young adolescent group than in the other groups, perhaps due to age differences in the hedonic perception of sweetness. These data are in agreement with human data on increased sensitivity to natural rewards during early adolescence (Crone and Dahl 2012; Van Leijenhorst et al. 2009).

Incubation of sucrose craving after adolescent- or adult-onset self-administration training

We found that the magnitude of incubation of sucrose craving was inversely related to the age of initiation of sucrose selfadministration with no evidence for incubation in the young adolescent training group. The lack of incubation in the young adolescent group is due in part to somewhat higher (albeit not statistically significant) cue-induced sucrose seeking on abstinence day 1 (Fig. 2). We believe it is unlikely that the age differences in incubation of sucrose craving are due to the observed age differences in sucrose intake during training. This is because no significant age group differences in cue responding were observed on abstinence day 1. Additionally, it is difficult to interpret the training data in reference to reward sensitivity, because while lever responding and total sucrose intake were higher in adult rats, sucrose intake per body weight was higher in the adolescent rats.

Our data are in agreement with results demonstrating that home-cage sucrose overconsumption during adolescence reduces the motivation to self-administer saccharin in adulthood (Vendruscolo et al. 2010). Our data are also in agreement with a previous report of $\mathrm{Li}$ and Frantz that the magnitude of incubation of cocaine craving is lower following adolescentonset self-administration training (P35) than following adultonset cocaine training ( $\mathrm{Li}$ and Frantz 2009). However, our results are different from a follow-up study of $\mathrm{Li}$ and Frantz (2010) reporting a lack of age differences in incubation of sucrose craving.

Methodological and procedural differences between our study and the study of Li and Frantz (2010) might account for the different results. These include the type of sucrose reward (sucrose pellet versus sucrose solution), duration of daily training session ( 30 min versus $3 \mathrm{~h}$ ) and the number of training days (14 versus 10). Another difference is the use of a within-session extinction-reinstatement procedure (six 30min operant extinction sessions without the discrete cues that 
a

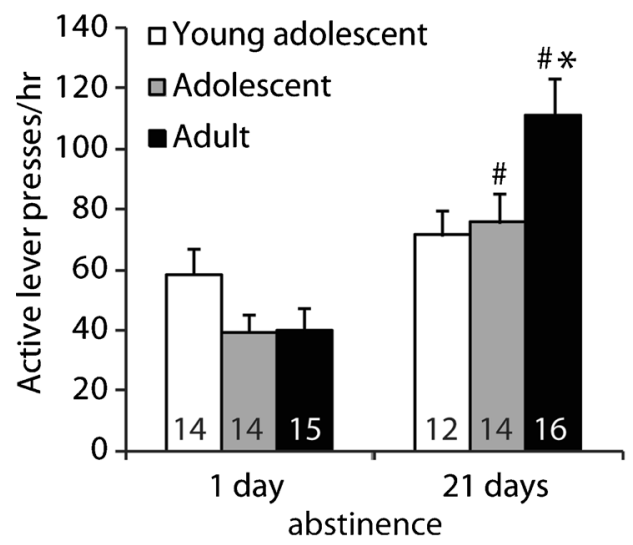

Fig. 2 Cue-induced sucrose seeking after 1 day or 21 days of abstinence. Number of active (a) and inactive (b) lever presses during the 1-h extinction session that was used to assess cue-induced sucrose seeking. During testing, lever presses led to contingent presentation of a tone-light

were followed by a 30-min cue-induced reinstatement test) in the Li and Frantz study versus a single 3-h extinction test in the presence of the discrete cue in our study. We suspect that the main reason for the difference between our findings and those of Li and Frantz is the use of longer training sessions and an oral sucrose solution that resulted in a significantly stronger magnitude of incubation of sucrose craving (about $300 \%$ increase in lever presses from abstinence day 1 to 21 in the adult rats) in our study versus their study (less than $50 \%$ increase in lever presses from abstinence day 1 to 30 in adult rats). In other words, the experimental procedure used in our study had more statistical power to detect age differences in incubation of sucrose craving. In conclusion, exposure to natural rewards may induce "stronger" incubation of reward craving in more mature rats than in early adolescent rats. It is currently unknown whether these results translate to the human situation, because while incubation of drug (nicotine or methamphetamine) craving has been demonstrated in human drug addicts (Bedi et al. 2011; Wang et al. 2013), incubation of sucrose or palatable food craving in humans was not assessed.

Role of nucleus accumbens synaptic plasticity in incubation of reward craving

We found that decreased AMPA/NMDA ratio on abstinence day 21 , but not day 1-3, was age dependent, with most pronounced changes in the adult training group, moderate changes the adolescent- training group, and very weak changes in the young adolescent training group. Changes in AMPA/NMDA ratio (a measure of postsynaptic changes in synaptic strength) are often used to infer whether long-term potentiation (LTP, increased AMPA/NMDA ratio) or longterm depression (LTD, decreased AMPA/NMDA ratio) had

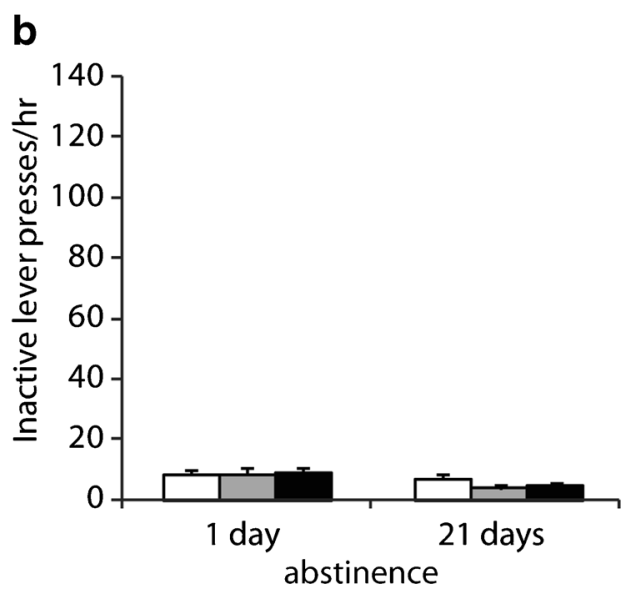

cue previously paired with sucrose reward but not sucrose. The numbers indicate number of rats per group. ${ }^{*} P<0.05$, different from both adolescent groups. $\# p<0.05$, different from day 1 within each age group

occurred in postsynaptic glutamatergic synapses (Citri and Malenka 2008; Ungless et al. 2001).

A consistent finding in the drug addiction literature is that prolonged withdrawal from self-administered or noncontingent cocaine ( $>3$ weeks) is associated with increased glutamate postsynaptic strength as indicated by increased AMPA/ NMDA ratio and other electrophysiology measures like intrinsic excitability and spontaneous excitatory postsynaptic currents (sEPSC) (Bowers et al. 2010; Thomas et al. 2008; Wolf and Ferrario 2010). Most relevant to our study is the evidence that enhanced glutamate postsynaptic synaptic strength due to accumulation of CP-AMPARs in NAc is not only associated with cue-induced cocaine seeking after prolonged withdrawal ( $>30$ days) from cocaine self-administration (Mameli et al. 2009) but also mediate enhanced (incubated) cue-induced cocaine seeking (Conrad et al. 2008; Loweth et al. 2013). Additionally, in vivo extracellular multineuronal recording results indicate that incubation of cocaine craving is associated with increased NAc core response to cocaine cues (Hollander and Carelli 2007). Our results indicate that sucrose reward induces a decrease in glutamate postsynaptic strength, suggesting that the plasticity mechanisms involved differ from those induced by cocaine and potentially other stimulant drugs.

Our observation that incubation of sucrose craving is associated with decreased AMPA/NMDA ratio was unexpected. Incubation of reward craving has been shown to occur with both drug- and nondrug rewards (Lu et al. 2004b; Pickens et al. 2011), and the neuronal mechanisms of drug and nondrug reward have been proposed to overlap (Kelley and Berridge 2002). Furthermore, relapse to drug and nondrug seeking is also thought to involve similar mechanisms (Calu et al. 2013; Nair et al. 2009). Our finding suggests that the mechanisms of incubation of sucrose craving and cocaine craving are dissociable and are likely linked to different forms of plasticity in the NAc. 
a

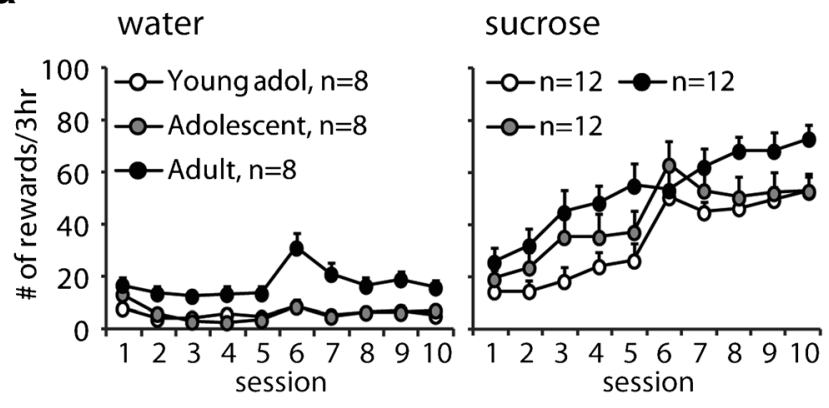

C

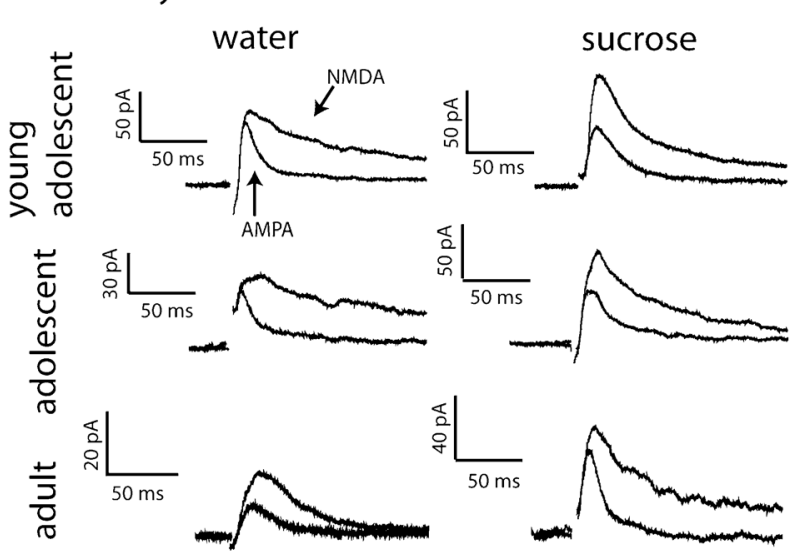

f

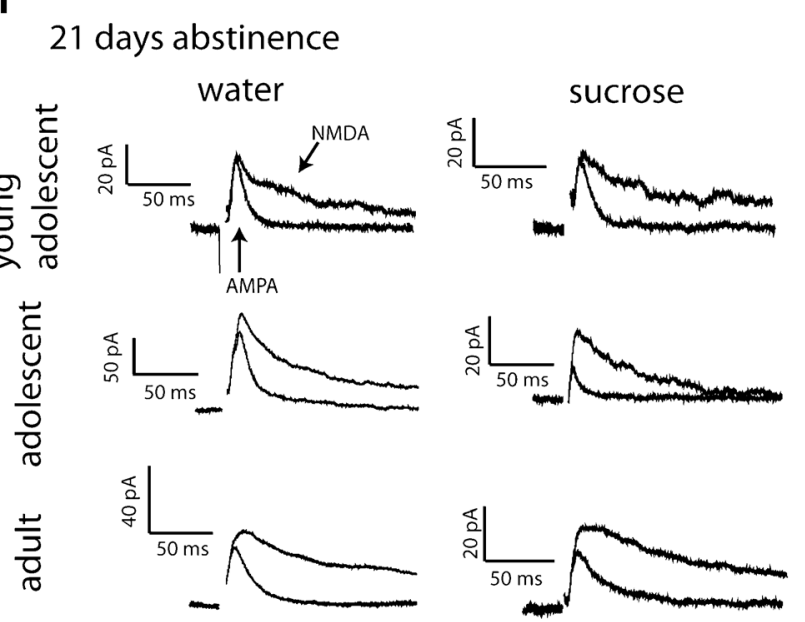

Fig. 3 AMPA/NMDA ratios in the nucleus accumbens core after 13 days or 3 weeks of abstinence from sucrose or water self-administration. a Mean \pm SEM number of earned rewards in rats trained to self-administer water (left) or sucrose (right) during the training phase. Brains were taken after 1-3 days or 21 days of abstinence without testing for cueinduced reward seeking. b Schematic of a parasagittal section through the nucleus accumbens core illustrating the relative placement of the stimulating electrode (bolt symbol) and recorded Neurobiotin-stained medium spiny neuron (inset) with respect to the forceps minor ( $f m i$ ) and anterior commissure $(a c a)$. c Representative traces of medium spiny neurons held at $+40 \mathrm{mV}$ in the absence (both AMPA and NMDA) and presence (only AMPA) of the NMDAR antagonist AP5 in rats after 1-3 days of abstinence from self-administered water or sucrose at the three different age

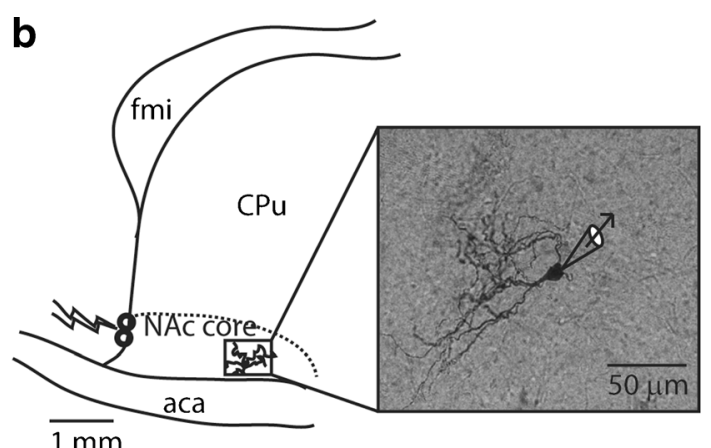

d

e
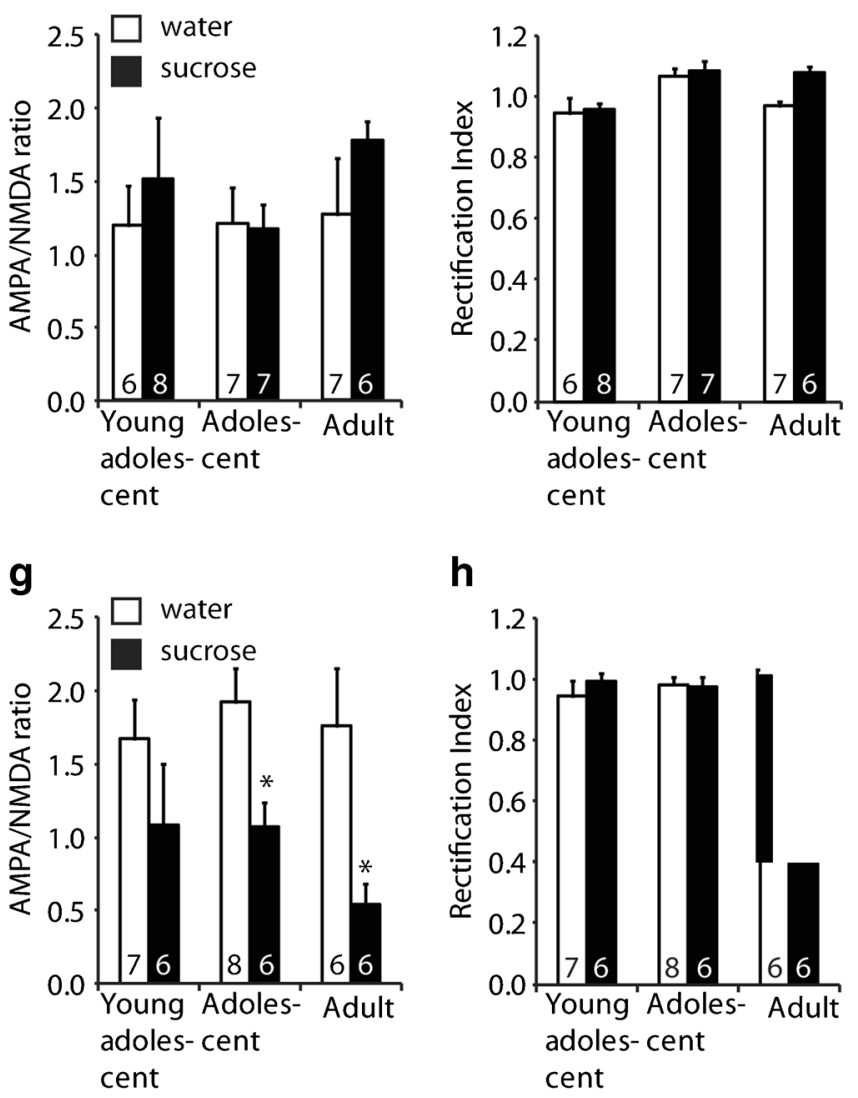

groups. Stimulation artifacts were removed for clarity. d Mean \pm SEM AMPA/NMDA in NAc after 1-3 days of abstinence from self-administered water (white bars) or sucrose (black bars). The numbers indicate cells per group ( $n=4-6$ rats per group). No more than two cells per rat were used. e Rectification index after 1-3 abstinence days. f Representative traces of medium spiny neurons after 3 weeks of abstinence from water or sucrose self-administration in the three different age groups. $\mathbf{g}$ Mean \pm SEM AMPA/NMDA ratio in NAc MSNs after 21 days of abstinence. The numbers indicate cells per group ( $n=4-6$ rats per group). No more than two cells per rat were used. $\mathbf{h}$ Rectification index after 21 abstinence days. ${ }^{*} p<0.05$, different from the water condition within each age group 
The notion that dissociable synaptic plasticity mechanisms mediate incubation of craving of cocaine versus sucrose is supported by several lines of indirect evidence. First, similar to our findings, Pitchers et al. found that NAc AMPA/NMDA ratio was decreased for up to 1 month after rewarding sexual experience (Pitchers et al. 2012). Additionally, while incubation of cocaine craving is associated with delayed long-lasting neuroadaptations (up to 90 days) in NAc (glutamate receptor expression, PKA activity, BDNF protein expression), no such neuroadaptations are observed with sucrose-experienced rats (Grimm et al. 2003; Lu et al. 2003). Another indirect evidence that incubation of cocaine and sucrose craving are mediated by different mechanisms is that the expression of incubation of cocaine craving persists for at least 90 days after drug selfadministration while incubation of sucrose craving is no longer observed after 60 abstinence days (Lu et al. 2004b).

The mechanism underlying the profound decrease in AMPA/NMDA ratio 21 days after cessation of sucrose selfadministration in the adolescent- and adult-onset training groups are unknown. The decrease in AMPA/NMDA ratio can be due to decreased strength of AMPAR-mediated transmission, increased NMDAR-mediated transmission, or a combination of both. Our negative data with the inward rectification index rule out changes in CP-AMPARs as a potential mechanism; these negative data are somewhat in agreement with earlier data showing only a modest change in CPAMPARs in cocaine-trained rats after 21 withdrawal days (Conrad et al. 2008). However, a direct comparison between our study and the study of Conrad et al. is not straightforward, because they recorded from coronal sections through the NAc core where electrical stimulation activates inputs from several brain regions (i.e., prefrontal cortex, hippocampus, amygdala, and thalamus) (Calhoon and O'Donnell 2013). In contrast, in our parasagittal sections, we presumably only stimulated prefrontal cortical inputs to NAc core. One speculation is that the profound decrease in AMPA/NMDA ratio on abstinence day 21 is due to the formation of so-called "silent synapses." Silent excitatory synapses are immature synapses that express stable NMDA receptors with AMPARs that are either absent or highly labile; these synapses are formed during early developmental stages and subsequently "mature" into fully functional synapses by recruiting AMPARs (Isaac et al. 1997; Kerchner and Nicoll 2008). In the NAc, formation of silent synapses has been implicated in cocaine-induced psychomotor sensitization (Brown et al. 2011; Huang et al. 2009; Koya et al. 2012; Lee and Dong 2011), and more recently in incubation of cocaine craving (Lee et al. 2013). In the case of cocaine self-administration, silent synapses are only detected during early but not late withdrawal (Lee et al. 2013). Furthermore, during early but not late withdrawal from cocaine, a reduction in AMPA/NMDA ratio is associated with silent synapse formation (Koya et al. 2012; Lee and Dong 2011). Therefore, a question for future research is whether sucrose self-administration leads to long-lasting formation of silent synapses in NAc.

\section{Concluding remarks}

We found that incubation of sucrose craving is critically dependent on the age of initial exposure to sucrose and that prolonged abstinence from sucrose causes a profound reduction in glutamate-mediated synaptic strength in NAc. The latter finding is opposite to the synaptic plasticity changes observed in this brain region after prolonged withdrawal from cocaine. While the mechanistic connection between incubation of sucrose craving and NAc synaptic plasticity changes are unknown, our data suggest that synaptic plasticity mechanisms of incubation of cocaine and sucrose craving are dissociable. This possibility is in agreement with recent results demonstrating that the neuronal mechanisms of incubation of craving across drug classes appear drug-specific, with some notable differences between heroin (Airavaara et al. 2011; Theberge et al. 2012) versus cocaine (Grimm et al. 2003; Lu et al. 2004a; Lu et al. 2009). The age-dependent changes in both the decrease in AMPA/NMDA ratio and the incubation of sucrose craving were unexpected and should be further explored in future studies.

Acknowledgments The research and writing of this paper was supported by extramural (R01 DA014020, PO'D) and intramural (YS) funds from NIDA, NIH.

Open Access This article is distributed under the terms of the Creative Commons Attribution License which permits any use, distribution, and reproduction in any medium, provided the original author(s) and the source are credited.

\section{References}

Airavaara M, Pickens CL, Stern AL, Wihbey KA, Harvey BK, Bossert JM, Liu QR, Hoffer BJ, Shaham Y (2011) Endogenous GDNF in ventral tegmental area and nucleus accumbens does not play a role in the incubation of heroin craving. Addiction Biol 16:261-272

Bedi G, Preston KL, Epstein DH, Heishman SJ, Marrone GF, Shaham Y, de Wit H (2011) Incubation of cue-induced cigarette craving during abstinence in human smokers. Biol Psychiatry 69:708-711

Blakemore SJ, Robbins TW (2012) Decision-making in the adolescent brain. Nat Neurosci 15:1184-1191

Bowers MS, Chen BT, Bonci A (2010) AMPA receptor synaptic plasticity induced by psychostimulants: the past, present, and therapeutic future. Neuron 67:11-24

Brenhouse HC, Sonntag KC, Andersen SL (2008) Transient D1 dopamine receptor expression on prefrontal cortex projection neurons: relationship to enhanced motivational salience of drug cues in adolescence. J Neurosci 28:2375-2382

Brown TE, Lee BR, Mu P, Ferguson D, Dietz D, Ohnishi YN, Lin Y, Suska A, Ishikawa M, Huang YH, Shen H, Kalivas PW, Sorg BA, Zukin RS, Nestler EJ, Dong Y, Schluter OM (2011) A silent synapse-based mechanism for cocaine-induced locomotor sensitization. J Neurosci 31:8163-8174 
Calhoon GG, O'Donnell P (2013) Closing the gate in the limbic striatum: prefrontal suppression of hippocampal and thalamic inputs. Neuron 78:181-190

Calu DJ, Chen YW, Kawa AB, Nair SG, Shaham Y (2013) The use of the reinstatement model to study relapse to palatable food seeking during dieting. Neuropharmacology

Casey BJ, Duhoux S, Malter Cohen M (2010) Adolescence: what do transmission, transition, and translation have to do with it? Neuron 67:749-760

Citri A, Malenka RC (2008) Synaptic plasticity: multiple forms, functions, and mechanisms. Neuropsychopharmacology 33:18-41

Conrad KL, Tseng KY, Uejima JL, Reimers JM, Heng LJ, Shaham Y, Marinelli M, Wolf ME (2008) Formation of accumbens GluR2lacking AMPA receptors mediates incubation of cocaine craving. Nature 454:118-121

Counotte DS, Smit AB, Pattij T, Spijker S (2011) Development of the motivational system during adolescence, and its sensitivity to disruption by nicotine. Developmental Cognitive Neuroscience 1:430-443

Crone EA, Dahl RE (2012) Understanding adolescence as a period of social-affective engagement and goal flexibility. Nat Rev Neurosci 13:636-650

Doherty JM, Cooke BM, Frantz KJ (2013) A role for the prefrontal cortex in heroin-seeking after forced abstinence by adult male rats but not adolescents. Neuropsychopharmacology 38:446-454

Everitt BJ, Robbins TW (2005) Neural systems of reinforcement for drug addiction: from actions to habits to compulsion. Nat Neurosci 8:14811489

Giedd JN (2004) Structural magnetic resonance imaging of the adolescent brain. Ann N Y Acad Sci 1021:77-85

Grimm JW, Hope BT, Wise RA, Shaham Y (2001) Neuroadaptation. Incubation of cocaine craving after withdrawal. Nature 412:141-142

Grimm JW, Shaham Y, Hope BT (2002) Effect of cocaine and sucrose withdrawal period on extinction behavior, cue-induced reinstatement, and protein levels of the dopamine transporter and tyrosine hydroxylase in limbic and cortical areas in rats. Behav Pharmacol 13:379-388

Grimm JW, Lu L, Hayashi T, Hope BT, Su TP, Shaham Y (2003) Timedependent increases in brain-derived neurotrophic factor protein levels within the mesolimbic dopamine system after withdrawal from cocaine: implications for incubation of cocaine craving. $\mathrm{J}$ Neurosci 23:742-747

Grimm JW, Fyall AM, Osincup DP (2005) Incubation of sucrose craving: effects of reduced training and sucrose pre-loading. Physiol Behav 84:73-79

Grimm JW, Manaois M, Osincup D, Wells B, Buse C (2007) Naloxone attenuates incubated sucrose craving in rats. Psychopharmacology (Berl) 194:537-544

Grimm JW, Harkness JH, Ratliff C, Barnes J, North K, Collins S (2011) Effects of systemic or nucleus accumbens-directed dopamine D1 receptor antagonism on sucrose seeking in rats. Psychopharmacology(Berl) 216:219-233

Gruber AJ, Powell EM, O'Donnell P (2009) Cortically activated interneurons shape spatial aspects of cortico-accumbens processing. $J$ Neurophysiol 101:1876-1882

Hollander JA, Carelli RM (2007) Cocaine-associated stimuli increase cocaine seeking and activate accumbens core neurons after abstinence. J Neurosci 27:3535-3539

Huang YH, Lin Y, Mu P, Lee BR, Brown TE, Wayman G, Marie H, Liu W, Yan Z, Sorg BA, Schluter OM, Zukin RS, Dong Y (2009) In vivo cocaine experience generates silent synapses. Neuron 63:40-47

Isaac JT, Crair MC, Nicoll RA, Malenka RC (1997) Silent synapses during development of thalamocortical inputs. Neuron 18:269-280

Karlsson RM, Kircher DM, Shaham Y, O'Donnell P (2013) Exaggerated cue-induced reinstatement of cocaine seeking but not incubation of cocaine craving in a developmental rat model of schizophrenia. Psychopharmacology (Berl) 226:45-51
Kelley AE, Berridge KC (2002) The neuroscience of natural rewards: relevance to addictive drugs. J Neurosci 22:3306-3311

Kerchner GA, Nicoll RA (2008) Silent synapses and the emergence of a postsynaptic mechanism for LTP. Nat Rev Neurosci 9:813-825

Koya E, Cruz FC, Ator R, Golden SA, Hoffman AF, Lupica CR, Hope BT (2012) Silent synapses in selectively activated nucleus accumbens neurons following cocaine sensitization. Nat Neurosci 15:1556-1562

Lee BR, Dong Y (2011) Cocaine-induced metaplasticity in the nucleus accumbens: silent synapse and beyond. Neuropharmacology 61: 1060-1069

Lee BR, Y. M, Huang YH, Wang X, Otaka M, Ishikawa M, Brown TE, Suska A, Lobo MK, Wolf ME, Nestler EJ, Shaham Y, Schlüter OM, Dong Y (2013) Silent synapse-mediated reorganization of the glutamatergic projection from amygdala to nucleus accumbens is critical to incubation of cocaine craving. Nature Neuroscience. doi:10. $1038 / \mathrm{nn} .3533$

Li C, Frantz KJ (2009) Attenuated incubation of cocaine seeking in male rats trained to self-administer cocaine during periadolescence. Psychopharmacology (Berl) 204:725-733

Li C, Frantz KJ (2010) Time-dependent increases in cue-induced reinstatement of sucrose seeking after sucrose self-administration in adolescence. Behav Brain Res 213:109-112

Loweth JA, Tseng KY, Wolf ME (2013) Adaptations in AMPA receptor transmission in the nucleus accumbens contributing to incubation of cocaine craving. Neuropharmacology. doi:10.1016/j.neuropharm. 2013.04.061

Lu L, Grimm JW, Shaham Y, Hope BT (2003) Molecular neuroadaptations in the accumbens and ventral tegmental area during the first 90 days of forced abstinence from cocaine selfadministration in rats. J Neurochem 85:1604-1613

Lu L, Dempsey J, Liu SY, Bossert JM, Shaham Y (2004a) A single infusion of brain-derived neurotrophic factor into the ventral tegmental area induces long-lasting potentiation of cocaine seeking after withdrawal. J Neurosci 24:1604-1611

Lu L, Grimm JW, Hope BT, Shaham Y (2004b) Incubation of cocaine craving after withdrawal: a review of preclinical data. Neuropharmacology 47(Suppl 1):214-226

Lu L, Hope BT, Dempsey J, Liu SY, Bossert JM, Shaham Y (2005) Central amygdala ERK signaling pathway is critical to incubation of cocaine craving. Nat Neurosci 8:212-219

Lu L, Wang X, Wu P, Xu C, Zhao M, Morales M, Harvey BK, Hoffer BJ, Shaham Y (2009) Role of ventral tegmental area glial cell linederived neurotrophic factor in incubation of cocaine craving. Biol Psychiatry 66:137-145

Mameli M, Halbout B, Creton C, Engblom D, Parkitna JR, Spanagel R, Luscher C (2009) Cocaine-evoked synaptic plasticity: persistence in the VTA triggers adaptations in the NAc. Nat Neurosci 12:1036-1041

Marchant NJ, Li X, Shaham Y (2013) Recent developments in animal models of drug relapse. Curr Opin Neurobiol. doi:10.1016/j.conb. 2013.01.003

Nair SG, Adams-Deutsch T, Epstein DH, Shaham Y (2009) The neuropharmacology of relapse to food seeking: methodology, main findings, and comparison with relapse to drug seeking. Prog Neurobiol 89:18-45

Neisewander JL, Baker DA, Fuchs RA, Tran-Nguyen LT, Palmer A, Marshall JF (2000) Fos protein expression and cocaine-seeking behavior in rats after exposure to a cocaine self-administration environment. J Neurosci 20:798-805

Palmer RH, Young SE, Hopfer CJ, Corley RP, Stallings MC, Crowley TJ, Hewitt JK (2009) Developmental epidemiology of drug use and abuse in adolescence and young adulthood: Evidence of generalized risk. Drug Alcohol Depend 102:78-87

Pickens CL, Airavaara M, Theberge F, Fanous S, Hope BT, Shaham Y (2011) Neurobiology of the incubation of drug craving. Trends Neurosci 34:411-420

Pitchers KK, Schmid S, Di Sebastiano AR, Wang X, Laviolette SR, Lehman MN, Coolen LM (2012) Natural reward experience alters 
AMPA and NMDA receptor distribution and function in the nucleus accumbens. PLoS One 7:e34700

See RE (2002) Neural substrates of conditioned-cued relapse to drug-seeking behavior. Pharmacol Biochem Behav 71:517529

Shalev U, Morales M, Hope B, Yap J, Shaham Y (2001) Timedependent changes in extinction behavior and stress-induced reinstatement of drug seeking following withdrawal from heroin in rats. Psychopharmacology (Berl) 156:98-107

Theberge FR, Pickens CL, Goldart E, Fanous S, Hope BT, Liu QR, Shaham Y (2012) Association of time-dependent changes in mu opioid receptor mRNA, but not BDNF, TrkB, or MeCP2 mRNA and protein expression in the rat nucleus accumbens with incubation of heroin craving. Psychopharmacology (Berl) 224:559571

Theberge FR, Li X, Kambhampati S, Pickens CL, St Laurent R, Bossert JM, Baumann MH, Hutchinson MR, Rice KC, Watkins LR, Shaham Y (2013) Effect of chronic delivery of the Toll-like receptor 4 antagonist $(+)$-naltrexone on incubation of heroin craving. Biol Psychiatry 73:729-737

Thomas MJ, Kalivas PW, Shaham Y (2008) Neuroplasticity in the mesolimbic dopamine system and cocaine addiction. $\mathrm{Br} \mathrm{J}$ Pharmacol 154:327-342
Tseng KY, O'Donnell P (2007) Dopamine modulation of prefrontal cortical interneurons changes during adolescence. Cereb Cortex $17: 1235-1240$

Uejima JL, Bossert JM, Poles GC, Lu L (2007) Systemic and central amygdala injections of the mGluR2/3 agonist LY379268 attenuate the expression of incubation of sucrose craving in rats. Behav Brain Res 181:292-296

Ungless MA, Whistler JL, Malenka RC, Bonci A (2001) Single cocaine exposure in vivo induces long-term potentiation in dopamine neurons. Nature 411:583-587

Van Leijenhorst L, Zanolie K, Van Meel CS, Westenberg PM, Rombouts SA, Crone EA (2010) What motivates the adolescent? Brain regions mediating reward sensitivity across adolescence. Cereb Cortex 20(1):61-69

Vendruscolo LF, Gueye AB, Darnaudery M, Ahmed SH, Cador M (2010) Sugar overconsumption during adolescence selectively alters motivation and reward function in adult rats. PLoS One 5:e9296

Wang G, Shi J, Chen N, Xu L, Li J, Li P, Sun Y, Lu L (2013) Effects of length of abstinence on decision-making and craving in methamphetamine abusers. PLoS One 8:e68791

Wolf ME, Ferrario CR (2010) AMPA receptor plasticity in the nucleus accumbens after repeated exposure to cocaine. Neurosci Biobehav Rev 35(2):185-211 